\title{
Information integration in price-quality tradeoffs: The effect of missing information
}

\author{
IRWIN P. LEVIN, RICHARD D. JOHNSON, and STEPHEN V. FARAONE \\ University of Iowa, Iowa City, Iowa
}

\begin{abstract}
Support for averaging processes in information integration has relied on experimental designs in which the amount of information to be integrated was varied. The usual interpretation is that subjects balance the values of the various stimuli presented on a given trial. An alternative interpretation is that subjects may use their perceptions of the relationship between stimulus dimensions to infer the value of missing information. In the present study, subjects were asked to rate their expected satisfaction with purchases of ground beef on the basis of quality and/or price information. The responses of some subjects appeared to be based on the inference that high prices imply high quality and low prices imply low quality when no quality information is given. Other subjects appeared to assign a constant value to missing information. An averaging model that incorporates inferred as well as presented stimulus values is proposed.
\end{abstract}

Averaging models of information integration assume that, in making a particular judgment or decision, subjects average, or balance, the values of the various stimuli presented. Empirically, averaging processes are identified by showing that the effect of a particular stimulus decreases as the number of other dimensions with which it is combined increases (Anderson, 1981). Support has been provided in situations as varied as personalityimpression formation (Anderson, 1965), attitude formation (Anderson, 1971), psychophysical and intuitive statistical judgments (Levin, 1975), clinical assessments (Anderson, 1972), evaluating student performance (Levin, Ims, Simpson, \& Kim, 1977), and consumer judgments (Troutman \& Shanteau, 1976).

There is, however, an altemative interpretation of the interaction between the effect of a particular stimulus dimension and the number of dimensions to be integrated. When subjects judge some situations that have less information than other situations, they may first infer the values of missing information by assuming specific interdimensional relationships and then integrate the inferred values with the presented values (Huber \& McCann, 1982; Yamagishi \& Hill, 1981).

Yamagishi and Hill (1981) developed a formal model based on these assumptions about the inferring of missing values. They called their model a "path-analytic" model because the net effect of this inference was assumed to depend on the direction of relationship among stimulus dimensions and the direction of relationship between each stimulus dimension and the overall judgment. They showed that for situations in which the product of

The authors wish to thank Linda Hanson for her assistance in collecting and compiling data. The authors' mailing address is: Department of Psychology, University of Iowa, Iowa City, Iowa 52242 . the set of correlations is positive (e.g., two stimulus dimensions that are each positively related to the overall judgment and positively related to each other), their model can account for the data previously interpreted as providing support for the averaging model, and for situ. ations in which the product is negative (e.g., two stimulus dimensions that are positively related to each other, but one stimulus dimension is positively related to the overall judgment and the other is negatively related to the overall judgment), their model generates predictions contrary to the averaging model. (See Figures 1-3 of their paper.) A similar model based on the directional effect of inferences on product evaluation was proposed by Huber and McCann (1982).

In order to understand these models, suppose, for example, that past experience has led subjects to assume a positive relationship between two stimulus dimensions that each have an increasing monotonic relationship with the response dimension. If subjects use this stored relationship to infer the value of missing information and add it to the impression response, then the effect of presenting a single piece of information will be increased because the net effect is like that of having presented two pieces of information of similar value. The results will then look like those predicted by an averaging model. If, on the other hand, a negative relationship is assumed by the subject in inferring the value of missing information, then the effect of a single piece of information will be neutralized and this will produce results contrary to those predicted by an averaging model.

The present paper addresses the following issue: Un der what conditions does the averaging model, as originally formulated, account for information integration, and under what conditions must an inferring-missing. values account be introduced? The issue is a fundamental one for understanding human judgment and decision 
making. It is also important, from a practical viewpoint, to know under which circumstances information presented to subjects is acted upon solely on the basis of its intrinsic value and under which circumstances subjects use prior associations to go beyond the information pre sented and infer other values. For example, will consumers assume that a new lower priced brand must be infe. rior in quality to older brands?

Because the majority of studies providing support for an averaging model of affective judgment have employed information dimensions that subjects are apt to assume to be positively related to each other and to the judg. ment (e.g., personality-trait information in person perception), the key to addressing this issue is to introduce tasks containing negative relationships. In two preliminary studies, negative relationships were introduced through instructional manipulations and through exposure to negatively correlated stimulus pairs. In the main study, a "price-quality tradeoff" task that relied on a well-established inverse relationship between stimulus dimensions was employed.

\section{Preliminary Studies}

In a pair of preliminary experiments, Levin, Johnson, Faraone, and Hanson (1982) varied the direction of relationship between stimulus dimensions and examined differences in the tendency to infer missing values. In one experiment, subjects were asked to rate their personal satisfaction with each of a series of economic situations defined by an annual rate of increase in housing costs and an annual rate of increase in energy and transportation costs, or by only one of these values. The nature of the relationship between the cost categories was manipulated in the instructions given to subjects. In the positiverelationship condition, subjects were told that increases in energy and transportation costs lead to increases in housing costs because they affect builders' costs. In the negative-relationship condition, subjects were told that increases in energy and transportation costs result in less money for consumers to spend on housing and that this drives down housing bids, which may lead to lower prices.

A "manipulation check" was included by providing trials in which only one cost category was presented and subjects had to fill in an expected value for the other category. Only those subjects whose responses on these trials conformed to their instructional condition $(n=70)$ were retained for the main data analyses. These analyses showed no systematic differences between instructional conditions in rating responses for stimulus pairs and single stimuli. Averaging was supported in each condition by showing that the same range of stimulus values had a greater effect on trials with single stimuli than on trials with stimulus pairs.

Similar results were obtained in a second experiment $(n=72)$, in which the direction of relationship was ma. nipulated through exposure to prior pairings of values of two stimulus dimensions. A and $B$ were said to be two different economic indicators, each of which varied over time periods. Each A-B pair was said to represent the val. ues of these indicators for a given time period. Some subjects were presented paired values that were generated from a positive relationship between $A$ and $B$, and some subjects who presented paired values generated from a negative relationship. Almost all subjects were able to correctly identify the nature of the relationship to which they had been exposed. However, results did not differ significantly across experimental conditions. Support for the averaging model was found for both positive- and negative-relationship conditions.

Taken together, these two preliminary studies, which included both positive and negative relationships between stimulus dimensions, provided support for an averaging model without having to assume that subjects infer the value of missing information. However, associations between stimulus dimensions were introduced experimentally and may not have been of sufficient strength and duration to promote the type of inference predicted by Huber and McCann (1982) and Yamagishi and Hill (1981).

\section{Main Study}

The main study reported in this paper was closer in design to the studies reported by Huber and McCann (1982) and Yamagishi and Hill (1981) than were the preliminary studies that failed to support the inferential model. Each of these reported studies employed a pricequality tradeoff task. However, the present study differed from Yamagishi and Hill's in that the amount of information (price and quality vs. only one of these values) was varied within subjects in the present study rather than between subjects, as in their study. The present study differed from both the Huber and McCann study and the Yamagishi and Hill study in that the subjects in the present study were classified in terms of their use of singlestimulus cues and that separate analyses of the information-integration process for two distinct groups are provided. Finally, the present study differed from the others in that a representative, rather than an orthogonal, design was used for generating realistic price-quality combinations.

The main advantages of a within-subject design are that the context in which judgments are made is the same for each subject, individual differences in the use of different parts of the response scale are controlled for, and greater power is achieved. For these reasons, within-subject designs are the most commonly used in studies of information integration. The advantage of classifying subjects is to avoid the unnecessary assumption of a single process for all subjects. The use of subtle information-processing strategies such as inferring values of missing information may well differ across subjects. A key feature of the present study is the discovery of apparently different inference processes for different subjects.

The price-quality tradeoff task chosen for the main study can be characterized as providing strong prior as- 
sociations that contribute to a negative product for the set of correlations among the two stimulus dimensions and the judgment response. (High quality, which is favorable, is associated with high price, which is unfavorable.) In conditions such as this, the path-analytic model and the averaging model make different predictions (Yamagishi \& Hill, 1981).

\section{METHOD}

Seventy-five students from introductory psychology courses at the University of lowa were asked to rate their expected satisfaction on a scale of 1 to 20 for each of a series of hypothetical purchases of ground beef. This type of purchase allows quantification of both the price and quality dimensions. Each purchase was described by the price per pound and the percentage of fat or by only one of these values. Values for single-stimulus trials were $\$ .80, \$ 1.00, \$ 1.20, \$ 1.40, \$ 1.60$, or $\$ 1.80$ per pound and $15 \%, 20 \%, 25 \%, 30 \%, 35 \%$, or $40 \%$ fat. Rather than pairing each price value with each quality value in a complete factorial design, a design was chosen for pairing values that was representative of an overall negative relationship between price per pound and percentage of fat, but that still included complete factorial subdesigns. Three separate $2 \times 2$ subdesigns were formed by: combining price-per-pound values of $\$ 1.80$ and $\$ 1.60$ with percentage-of-fat values of $15 \%$ and $20 \%$; combining price-perpound values of $\$ 1.40$ and $\$ 1.20$ with percentage-of-fat values of $25 \%$ and $30 \%$; and combining price-per-pound values of $\$ 1.00$ and $\$ .80$ with percentage-of-fat values of $35 \%$ and $40 \%$. These three subdesigns can be classified, respectively, as: high price, high quality; medium price, medium quality; and low price, low quality. (The complete design is illustrated in Figure 1.)

Two instructional conditions were formed. In Condition A ( $n=37$ ), the subjects were explicitly reminded that on some trials both price-per-pound and percentage-of-fat values would be presented, whereas on other trials only one of these values would be presented, and that on these latter trials they should base their responses on only that one piece of information. In Condition $\mathrm{B}(\mathrm{n}=38)$, no such reminder was given. In each condition, the subjects were allowed to work at their own pace but were instructed to examine all the situations in their booklet before beginning their ratings.

\section{RESULTS AND DISCUSSION}

Because responses did not differ systematically as a function of instructional condition, the data are combined across conditions in Figure 1. The left-hand panel plots these data with price per pound on the abscissa in order to compare responses to price-quality pairs with
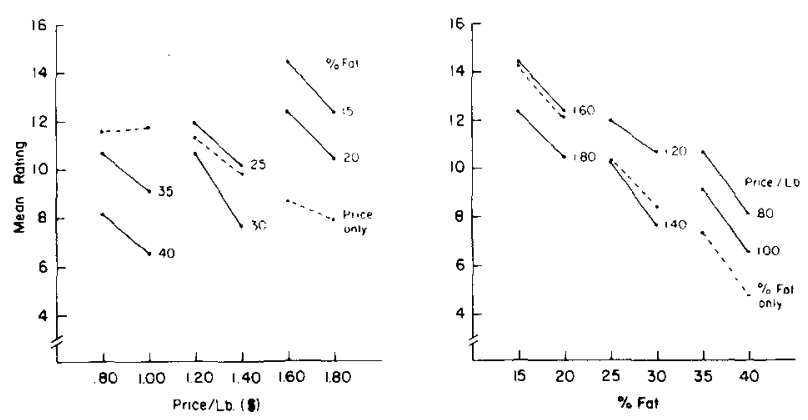

Figure 1. Mean ratings with price per pound on the abscissa (left panel) and percentage of fat on the abscissa (right panel). responses to price-only trials, and the right-hand panel plots the data with percentage of fat on the abscissa in order to compare price-quality pairs with qualityonly trials. Probably the most noticeable feature of Figure 1 is that ratings are highest for the high-price/highquality pairs and lowest for the low-price/low-quality pairs. This difference is statistically significant. It thus appears that, for the selected stimulus values, quality (percentage of fat) affected responses more than did price.

Theoretical interest centers around a comparison of the slopes of the dotted lines (single-stimulus trials) and the solid lines (stimulus pairs). An averaging model predicts that the dotted lines will be steeper than the solid lines because on paired trials each stimulus dimension is assumed to contribute only a proportion of its total possible effect. A path-analytic model predicts that the dotted lines will be flatter than the solid lines because on single stimulus trials the effect of a given favorable or unfavorable stimulus value is assumed to be neutralized by inferring an opposite value for the missing information.

Results in the left-hand panel clearly favor the pathanalytic model; the dotted lines are significantly flatter than the solid lines in five of six comparisons. The most ready interpretation of this finding is that subjects infer that high prices imply low percentages of fat and that low prices imply high percentages of fat, and that they use these inferred values in making their ratings. Note, however, that ratings for single-stimulus trials with high prices are considerably lower than ratings for high-price/ high-quality pairs, and ratings for single-stimulus trials with low prices are considerably higher than ratings for low-price/low-quality pairs. The stronger the inference effect, the more closely responses to these single stimuli should have resembled responses to the corresponding stimulus pairs. For example, the response to price per pound $=\$ .80$ (the lowest price) should have been similar to the response to price per pound $=\$ .80$, percentage of fat $=40 \%$ (the lowest price paired with the poorest quality). This was clearly not the case. Thus, the assumption that all subjects infer missing values based on the price-quality relationship and incorporate these in their impression responses does not completely explain the results.

In the right-hand panel of Figure 1, the dotted line is approximately parallel to the solid lines in each of the three subdesigns. None of the six slope comparisons between solid and dotted lines was statistically significant. This does not provide differential support for the averaging model or the path-analytic model.

Although Figure 1 provides a ready summary of group results, it masks some interesting individual differences. When responses to single-stimulus trials were observed on an individual-subject basis, two distinct types of responders could be identified. Generally, one would expect that, on single-stimulus trials, satisfaction ratings would decrease as a function of increases in both price 
per pound and percentage of fat. Although virtually all subjects showed the expected effect on the percentageof-fat dimension, a sizable minority of subjects showed deviations on the price-per-pound dimension. A total of 26 subjects showed either an increasing response function as price increased or a curvilinear function whereby intermediate price values were rated higher than low or high prices. These subjects seemed to infer strongly that low prices implied low quality and/or that high prices implied high quality.

The subjects were reclassified into two subgroups depending on their responses to price-only trials. Responses of the two subgroups are summarized in Figure 2. The upper panels show the responses of the Group 1 subjects $(n=26)$, who displayed a nondecreasing response function for price-only trials, and the lower panels show the responses of the Group 2 subjects $(n=42)$, for whom satisfaction ratings did decrease as price per pound increased on price-only trials. The proportion of subjects classified into Groups 1 and 2 did not differ significantly as a function of instructional condition; seven subjects displayed irregular response functions for price-only trials and were not included.

First, consider the left-hand panels of Figure 2. The difference between Groups 1 and 2 on price-only trials can be seen clearly; the slope of the dotted line was significantly different from that of the solid line in five of six comparisons for Group 1, but in only one of six comparisons for Group 2. Other differences are evident as well. For example, Group 1 showed more variation in responses across subdesigns than did Group 2. Group 1 had significantly higher ratings than Group 2 for highprice/high-quality pairs but significantly lower ratings than Group 2 for low-price/low-quality pairs. This can be interpreted to mean that the subjects in Group 1 gave relatively higher weight to quality (percentage of fat) and lower weight to price than did the subjects in Group 2. ${ }^{1}$ An additional difference between the groups
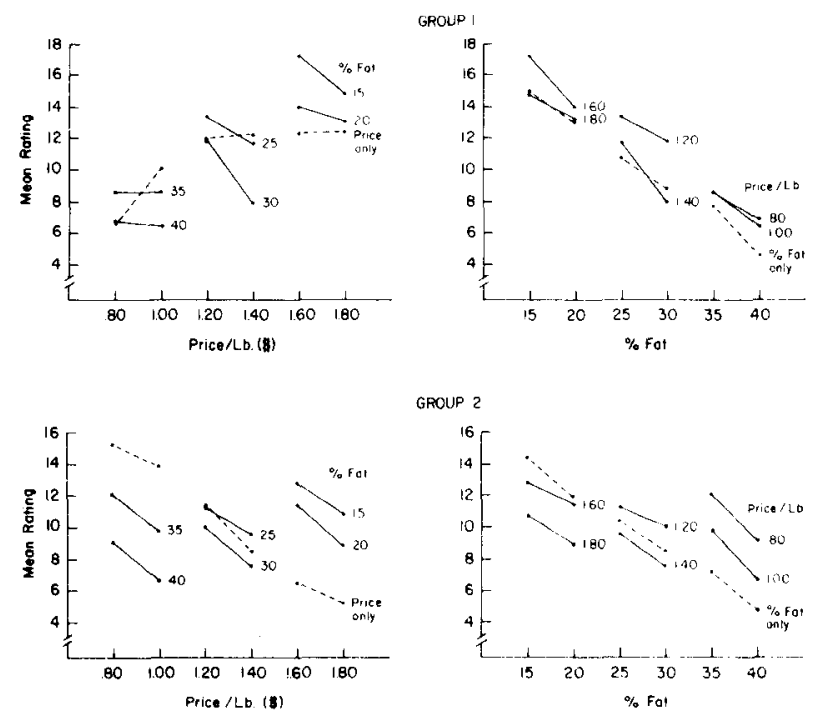

Figure 2. Mean ratings for subgroups with different response functions on price-only trials. is that the dotted lines are much closer in elevation to the solid lines for Group 1 than for Group 2 in the highprice/high-quality and low-price/low-quality subdesigns. This provides additional support that the subjects in Group 1 were inferring values on the quality dimension when only price information was presented.

The upper right-hand panel of Figure 2 shows that the subjects in Group 1 displayed a "conventional" response function for quality-only trials. Ratings decreased as percentage of fat increased on these trials. The dotted line in each subdesign is approximately parallel to the solid lines; five of the six slope comparisons were nonsignificant.

For subjects in Group 2, the dotted lines for singlestimulus trials in each panel are approximately parallel to the solid lines for stimulus pairs (only 1 of 12 slope differences was significant), but responses to singlestimulus trials are significantly higher than responses to price-quality pairs in the low-price/low-quality subdesign and responses to single-stimulus trials are significantly lower than responses to price-quality pairs in the highprice/high-quality subdesign. Because the responses to stimulus pairs were much different from the responses to single stimuli, it does not appear that the subjects in Group 2 were using the relationship between price and quality to infer missing values.

In summary, the current data suggest that the tendency to infer the value of missing information and incorporate inferred values into the impression response varies both between subjects and between information dimensions within subjects. The following section presents a formalized model to account for the differences in slope and elevation between the dotted and solid lines in the various panels of Figure 2.

\section{An Averaging Model of Inferred Values}

A simple form of the averaging model applied to judgments based on two stimulus dimensions would be as follows:

$$
R=\frac{W_{0} S_{0}+W_{1} S_{1}+W_{2} S_{2}}{W_{0}+W_{1}+W_{2}}
$$

where $R$ is the response, $W_{1}$ and $S_{1}$ are the weight and scale value, respectively, of the first stimulus dimension, $W_{2}$ and $S_{2}$ are the weight and scale value of the second stimulus dimension, and $W_{0}$ and $S_{0}$ are the weight and scale value of the subject's initial opinion or response bias. Note that the effective weight of $S_{1}$ is $W_{1} /\left(W_{0}+\right.$ $\mathrm{W}_{1}+\mathrm{W}_{2}$ ).

If only $S_{1}$ is presented and the subject's responses are based on only that one stimulus dimension, then the model becomes

$$
\mathrm{R}=\frac{\mathrm{W}_{0} \mathrm{~S}_{0}+\mathrm{W}_{1} \mathrm{~S}_{1}}{\mathrm{~W}_{0}+\mathrm{W}_{1}}
$$


Note that the effective weight of $S_{1}$ here is $W_{1} /\left(W_{0}+W_{1}\right)$. If only $S_{1}$ is presented but the subject infers a value of $S_{2}$ from the value of $S_{1}$, then the model becomes

$$
\mathrm{R}=\frac{\mathrm{W}_{0} \mathrm{~S}_{0}+\mathrm{W}_{1} \mathrm{~S}_{1}+\mathrm{W}_{2}{ }^{\prime} \mathrm{S}_{2}{ }^{\prime}}{\mathrm{W}_{0}+\mathrm{W}_{1}+\mathrm{W}_{2}^{\prime}},
$$

where $\mathrm{W}_{2}{ }^{\prime}$ and $\mathrm{S}_{2}{ }^{\prime}$ are the weight and inferred scale value of the second stimulus dimension. A reasonable assumption is that the weight of an inferred value is no greater than the weight of a presented value, or $0 \leqslant$ $\mathrm{W}_{2}{ }^{\prime} \leqslant \mathrm{W}_{2}$.

Now let us apply Equation 3 to one of the factorial subdesigns, the low-price/low-quality subdesign, where $S_{1}$ is price per pound $=\$ .80$ or $\$ 1.00$ and $S_{2}$ is percentage of fat $=35 \%$ or $40 \%$. First, consider the case in which there is no inferring of missing values. Then, $\mathrm{W}_{2}{ }^{\prime}=0$ and Equation 3 reduces to Equation 2. This has two consequences: First, the dotted line for priceonly trials will have a steeper slope than will the solid lines for stimulus pairs, because $W_{1} /\left(W_{0}+W_{1}\right)>$ $\mathrm{W}_{1} /\left(\mathrm{W}_{0}+\mathrm{W}_{1}+\mathrm{W}_{2}\right)$; second, the dotted line will be higher in elevation than the solid lines, because low prices will not be neutralized by high inferred levels of percentage of fat.

Next, consider the possibility that subjects infer the value of a missing piece of information and assign it approximately the same weight as if that information had actually been presented (i.e., $W_{2}{ }^{\prime} \cong W_{2}$ ). Two cases need to be distinguished here. In the first case, subjects infer the value of a missing piece of information by assuming a perfect negative relationship between $S_{1}$ and $S_{2}$. In this case, the response to $S_{1}=\$ .80$ (the lowest price) would be similar to the response to the pair $\mathrm{S}_{1}=\$ .80, \mathrm{~S}_{2}=40 \%$ (the lowest price paired with the highest percentage of fat) and the response to $\mathrm{S}_{1}=\$ 1.00$ would be similar to the response to the pair $\mathrm{S}_{1}=\$ 1.00, \mathrm{~S}_{2}=35 \%$. The dotted line would then be less steep than (or even reversed in direction to) the solid lines, and the dotted line would be of approximately the same elevation as the average of the solid lines.

In the second case, subjects infer the value of a missing piece of information by assigning it a constant value (e.g., an approximately neutral value) that is independent of the value of the information presented. In this case, the response to $S_{1}=\$ .80$ would be higher than the response to the pair $S_{1}=\$ .80, S_{2}=40 \%$, because the inferred value of $S_{2}$ would not be as negative as that for $40 \%$, and by similar reasoning the response to $S_{1}=\$ 1.00$ would be higher than the response to the pair $S_{1}=\$ 1.00, S_{2}=35 \%$. The dotted line would then be higher than the solid lines but approximately parallel to them, because assuming a constant value of $S_{2}$ leaves the slope unchanged. These predictions are summarized in Table 1.

For levels of $W_{2}{ }^{\prime}$ that are between 0 and $W_{2}$ and for inferred values $\left(S_{2}{ }^{\prime}\right)$ that are regressed to a neutral value, results intermediate between those in the three extreme cases described in Table 1 can be expected. However, the various panels of Figure 2 can be readily differentiated in terms of their approximation to the extreme cases. The upper left-hand panel (Group 1) comes closest to the case in which subjects are assumed to assign values to missing quality information by inferring that favorable levels of one dimension go with unfavorable levels of the other dimension. The lower panels (Group 2) come closest to the case in which subjects are assumed to assign a constant value to the missing information. The greater difference in elevation between the solid and dotted lines in the lower lefthand panel than in the lower right-hand panel reflects the greater weight for quality than for price. Results in the upper right-hand panel show less evidence for the inferring of missing values, probably because subjects in Group 1 placed little importance on missing price information.

\section{Supplementary Data}

Support for Yamagishi and Hill's (1981) model of inferring missing values was strongest in the present study when those subjects who assigned especially high weight to the quality dimension were presented trials on which quality information was missing. Recalling that subjects were initially classified on the basis of their responses to trials with missing quality information, it is of interest to note that similar results were obtained when the relative importance of price and quality was manipulated experimentally.

Table 1

Predictions of Averaging Model for Specific Boundary Conditions

\begin{tabular}{lll}
\hline \multicolumn{1}{c}{ Condition } & \multicolumn{1}{c}{ Slope Comparison } & \multicolumn{1}{c}{ Elevation Comparison } \\
\hline No inferring of missing values. & $\begin{array}{l}\text { Slope for single-stimulus trials } \\
\text { will be greater than slope for } \\
\text { stimulus pairs. }\end{array}$ & $\begin{array}{l}\text { Single-stimulus trials will produce } \\
\text { more polarized responses than } \\
\text { stimulus pairs. }\end{array}$ \\
$\begin{array}{l}\text { Subjects infer a constant } \\
\text { (neutral) value for missing }\end{array}$ & $\begin{array}{l}\text { No difference in slope for } \\
\text { single-stimulus trials and } \\
\text { stimulus pairs. }\end{array}$ & $\begin{array}{l}\text { Single-stimulus trials will produce } \\
\text { more polarized responses than } \\
\text { stimulus pairs. }\end{array}$ \\
$\begin{array}{l}\text { Subjects infer the value of missing } \\
\text { information by assuming a }\end{array}$ & $\begin{array}{l}\text { Slope for single-stimulus trials } \\
\text { will be less than slope for } \\
\text { negative interstimulus relationship. }\end{array}$ & stimulus pairs.
\end{tabular}


Levin and Johnson (1982) used instructions to manipulate the relative importance of price and quality. The price group $(n=31)$ was told to consider the fact that, in making their meat purchases, they were on a very limited budget. The quality group $(\mathrm{n}=32)$ was told that they were on a very liberal budget, and the control group $(\mathrm{n}=27)$ was not told anything about a budget. As in the present study, subjects were instructed to rate their expected satisfaction with purchases of ground beef described by the price per pound and percentage of fat or by only one of these values.

The instructional manipulation was effective in changing the relative importance of price and quality. The price effect was greatest for the price group, and the quality effect was greatest for the quality group. Furthermore, two subject variables had significant effects. The effect of price was greatest for students from lowincome families and for those with the most responsibility for their own support. The effect of quality was greatest for students from high-income families and for those with the least responsibility for their own support. These differences tend to lend "face validity" to the responses.

Evidence for inferring missing values differed between groups. Only the quality group showed a smaller effect of price on price-only trials than on trials providing price-quality pairs. However, even this effect of using price information to infer inverse quality values when the importance of quality was stressed was small in magnitude.

\section{CONCLUSIONS}

Data from the preliminary studies provide support for an averaging model without the need to assume that subjects infer the value of missing information. For the negative-relationship conditions, this is contrary to the predictions of a path-analytic model. A possible explanation is that the negative relationships created in the laboratory tasks were not sufficiently strong to lead subjects to infer a favorable value of one variable when an unfavorable value of the other variable was presented, and vice versa. By contrast, the strong preexperimental negative relationship utilized in the price-quality tradeoff study did provide evidence that subjects can use stored information to infer missing values. Even here, however, an asymmetry that warrants further study was observed. Subjects were more apt to infer quality from price than they were to infer price from quality. (It is clearly in the best interest of the producer of a new lower priced brand to provide favorable information to counter the inference of low quality.)

Subjects appeared to differ in the strategies they used when information was missing. There was strong evidence for only about a third of the subjects that their responses when information about quality was missing were based on the inference that high prices imply high quality and/or that low prices imply low quality. These are the only subjects who provide strong support for Yamagishi and Hill's (1981) model. Other subjects appear to have assumed a constant value for missing information, rather than varying their inferences as a function of the stimulus values actually presented on a given trial. Other researchers report similar results. For example, Leon (1980) investigated the integration of intent and consequences on children's judgments of naughtiness. Ratings obtained on intent-only trials were consistent with the assumption that the children tended to infer a slightly negative consequence when no information about consequence was given. Anderson (1982) noted the importance of such findings for theory development by illustrating how averaging theory can provide novel evidence on inference processes.

We conclude that traditional averaging models provide a good description of how responses vary over stimulus combinations in a wide variety of informationintegration tasks. Furthermore, the balancing of overt stimulus values implied by an averaging model probably represents a good account of the information-integration process in many of these tasks. Nevertheless, the present results and those of other researchers indicate that there are situations in which the traditional averaging models fall short of providing a complete process analysis. Situations in which subjects infer the value of missing information may be more the exception than the rule-for example, inferences based on interstimulus relationships appear to occur only when the relationship between stimulus dimensions is strongly established and when the missing information is deemed crucial to the required judgment-but this should not detract from the importance of this concept for understanding behavior. An averaging-of-inferred-values model such as that proposed here represents one way of incorporating this interesting information-integration process into the framework of a traditional model that has received considerable prior support.

\section{REFERENCES}

Anderson, N. H. (1965). Averaging versus adding as a stimulus combination rule in impression formation. Journal of Experimental Psychology, 70, 394-400.

ANDERSON, N. H. (1971). Integration theory and attitude change. Psychological Review, 78, 171-206.

ANDERSon, N. H. (1972). Looking for configurality in clinical judgment. Psychological Bulletin, 78, 93-102.

ANDE RSON, N. H. (1981). Foundations of information integration theory. New York: Academic Press.

ANDERsON, N. H. (1982). Methods of information integration theory. New York: Academic Press.

Huber, J., \& McCanN, J. (1982). The impact of inferential beliefs on product evaluations. Journal of Marketing Research, 19, 324-333.

LEON, M. (1980). Integration of intent and consequence information in children's moral judgments. In F. Wilkening, J. Becker, \& T. Trabasso (Eds.), Information integration by children. Hillsdale, NJ: Erlbaum.

LEVIN, I. P. (1975). Information integration in numerical judg- 
ments and decision processes. Journal of Experimental Psychology: General, 104, 39-53.

Levin, I. P., Ims, J. R., Simpson, J. C., \& Kim, K. J. (1977). The processing of deviant information in prediction and evaluation. Memory \& Cognition, 5, 679-684.

Levin, I. P., \& Johnson, R. D. (1982, November). Information integration in price-quality tradeoffs: Effects of missing information. Paper presented at the meeting of the Psychonomic Society, Minneapolis.

Levin, I. P., Johnson, R. D., Faraone, S. V., \& Hanson, L. T. (1982, May). A veraging processes in the formation of economic attitudes. Paper presented at the meeting of the Midwestern Psychological Association, Minneapolis.

Troutman, C. M., \& Shanteau, J. (1976). Do consumers evaluate products by adding or averaging attribute information? Journal of Consumer Research, 3, 101-106.

YAMAgISHI, T., \& HiLl, C. T. (1981). Adding versus averaging models revisited: A test of a path-analytic integration model. Journal of Personality and Social Psychology, 41, 13-25.

\section{NOTE}

1. At the end of the experimental session, subjects were asked to estimate their family income and the percentage of their own support for which they were personally responsible. In no case did one of these subject variables enter into significant interactions with the variables manipulated in the experiment. However, some interesting trends were observed. Most in teresting of these was the tendency for the subjects in Group 1 that is, those who gave higher weight to quality and were most apt to infer quality values when only price information was presented-to report higher family income (mean $=\$ 50,700$ ) than the subjects in Group 2 (mean $=\$ 39,800)$.

(Manuscript received October 20,1982; revision accepted for publication September 26,1983 .) 